\title{
ON SPECTRAL PERMANENCE FOR CERTAIN BANACH ALGEBRAS
}

\author{
C. E. RICKART
}

Introduction. Let $\mathfrak{A}$ be a commutative Banach algebra which is algebraically embedded in a second such Banach algebra $\mathfrak{B}$. Let $\phi: x \rightarrow x(\phi)$ denote a homomorphism of $\mathfrak{A}$ into the complex numbers. The main problem considered here is that of extending $\phi$ to all of $\mathfrak{B}$. This extension is, of course, not always possible. On the other hand, it has been proved by Silov $[3 ; 12]$ that certain $\phi$ can be extended to $\mathfrak{B}$ in case the embedding of $\mathfrak{A}$ in $\mathfrak{B}$ is an isometry. It is proved below (Theorem 1) that the extension can always be made if $\mathfrak{A}$ is semisimple and regular in a sense defined by Silov [13]. This implies that elements of $\mathfrak{A}$ have the same spectra in $\mathfrak{A}$ as in $\mathfrak{B}$, and also has as a corollary a result due to Kaplansky [5, Theorem 6.2] concerning the minimal character of the norm in an algebra $C(\Omega)$. In $\$ 2$ some results for the noncommutative case are obtained when $\mathscr{U}$ is a $B^{*}$-algebra [9]. For example, if $\mathfrak{A}$ is a $B^{*}$-algebra which is algebraically embedded in an arbitrary Banach algebra $\mathfrak{B}$ and $x$ is an element of $\mathscr{A}$, then the spectrum of $x$ in $\mathfrak{A}$ is equal to the join of the spectrum of $x$ and the conjugate of the spectrum of $x^{*}$ in $\mathfrak{B}$. Also, the spectral radii of $x$ in $\mathfrak{A}$ and in $\mathfrak{B}$ are always equal. If in addition the involution in $\mathfrak{A}$ can be extended to $\mathfrak{B}$, then the spectra of $x$ in $\mathfrak{A}$ and in $\mathfrak{B}$ are equal.

1. Regular Banach algebras. Let $\mathfrak{A}$ be a real or complex commutative Banach algebra, not necessarily with an identity element. Let $\Phi_{\mathfrak{a}}$ be the set of all (algebra) homomorphisms of $\mathfrak{A}$ into the complex numbers. The zero homomorphism is included here and will be denoted by $\phi_{\infty}$. The image of $x \in \mathfrak{A}$ under $\phi \in \Phi_{\mathfrak{a}}$ is denoted by $x(\phi)$. If $\mathfrak{A}$ is complex, then every element of $\Phi_{\mathfrak{a}}$ different from $\phi_{\infty}$ maps $\mathfrak{A}$ onto the complex numbers. If $\mathfrak{A}$ is real, then an element of $\Phi_{\mathfrak{A}}$ may map $\mathfrak{A}$ onto either the reals or complexes. The kernel of any $\phi$ different from $\phi_{\infty}$ is a maximal regular ${ }^{1}$ ideal in $\mathfrak{A}$. Conversely, every maximal regular ideal in $\mathfrak{A}$ is the kernel of an element of $\Phi_{\mathfrak{a}}$ different from $\phi_{\infty}$. However, if $\mathfrak{A}$ is real, this correspondence between elements of $\Phi_{\mathfrak{n}}-\boldsymbol{\phi}_{\infty}$ and maximal regular ideals need not be one-to-one. ${ }^{2}$

In the usual way, $\Phi_{\mathfrak{r}}$ is made into a topological space via the funda-

Presented to the Society, April 26, 1952; received by the editors June 18, 1952.

${ }^{1}$ An ideal $I$ is regular provided there exists an element $e$ which is an identity modulo $I$; i.e., $e x-x$ belongs to $I$ for every $x$ [11].

${ }^{2}$ Consider, for example, a $\phi$ which maps $\mathscr{A}$ onto the complex numbers and define $\phi^{\prime}$ by $x\left(\phi^{\prime}\right)=\overline{x(\phi)}$. Then $\phi^{\prime} \in \Phi_{\mathfrak{q}}, \phi^{\prime} \neq \phi$, but $\phi$ and $\phi^{\prime}$ have the same kernel. 
mental system of neighborhoods

$N_{\phi}\left(a_{1}, \cdots, a_{n} ; \epsilon\right)=\left\{\phi^{\prime}|| a_{i}\left(\phi^{\prime}\right)-a_{i}(\phi) \mid<\epsilon, i=1, \cdots, n, \phi^{\prime} \in \Phi_{\mathfrak{A}}\right\}$,

where $\epsilon>0$ and $a_{1}, \cdots, a_{n}$ is any finite set of elements in $\mathfrak{A}$. Under this topology, $\Phi_{\mathfrak{r}}$ becomes a compact Hausdorff space called the structure space of $\mathfrak{A}$. If $\mathfrak{A}$ has an identity element, then $\phi_{\infty}$ is an isolated point of $\Phi_{\mathfrak{x}}$. If $\mathfrak{A}$ does not have an identity and $\mathfrak{A}^{\prime}$ is the algebra obtained in the usual way by adjoining to $\mathscr{U}$ an identity element, then it is easy to see that $\Phi_{\mathfrak{X}}$, can be obtained from $\Phi_{\mathfrak{Y}}$ by adding a single isolated point, the zero homomorphism of $\mathfrak{A}^{\prime}$. If $\mathfrak{A}$ is complex, then $\Phi_{\mathfrak{x}}-\phi_{\infty}$ can be identified with the space of maximal regular ideals of $\mathfrak{A}$ as usually defined. If $\mathfrak{A}$ is real and $\mathfrak{A} c$ is the complexification [5] of $\mathfrak{A}$, then $\Phi_{\mathfrak{Y}}$ and $\Phi_{\mathfrak{x}}$ are homeomorphic under the natural mapping from $\Phi_{\mathfrak{a}}$ to $\Phi_{\mathfrak{X}}$ obtained by restricting elements of $\Phi_{\mathfrak{X}}$ to $\mathfrak{A}$.

For each $x$ in $\mathfrak{A}, x(\phi)$ is a continuous function of $\phi$ which vanishes at the "point at infinity" $\phi_{\infty}$. The set of nonzero points in the range of $x(\phi)$ coincides with the set of nonzero points in the spectrum ${ }^{3}$ $\sigma_{\mathfrak{n}}(x)$. For the sake of simplicity, we shall include zero in the spectrum in all cases so that the range of $x(\phi)$ is always equal to $\sigma \mathfrak{q}(x)$. In any case, the spectral radius $r_{\mathfrak{x}}(x)$ is given by $\lim \left\|x^{n}\right\|^{1 / n}$ as $n \rightarrow \infty[6$, p. 818]. An element such that $x(\phi)=0$ for every $\phi$ is necessarily quasi-regular. Semi-simplicity of $\mathfrak{A}$ is equivalent to the condition that $x(\phi)=0$ for every $\phi$ imply $x=0$.

The algebra $\mathfrak{A}$ is called regular ${ }^{5}$ provided, for every closed set $F \subset \Phi_{\mathfrak{q}}$ and $\phi_{0} \in \Phi_{\mathfrak{X}}-F$, there exists $u \in \mathfrak{A}$ such that $u(\phi)$ is constant on $F$ with value different from $u\left(\phi_{0}\right)$. If $\mathfrak{A}$ is complex, then an equivalent definition is that, for every closed set $F \subset \Phi_{\mathfrak{x}}$ and $\phi_{0} \in \Phi_{\mathfrak{x}}-F$ with $\phi_{0} \neq \phi_{\infty}$, there exist $u \in \mathfrak{Y}$ such that $u\left(\phi_{0}\right)=1$ and $u(\phi)=0$ for $^{6} \phi \in F$. The latter definition is the one given by Silov [12] for complex $\mathfrak{A}$

${ }^{3}$ In defining the spectrum, we prefer the operation $x \circ y=x+y-x y$ used by Segal [11] and Hille [4, p. 455] rather than $x+y+x y$ used by some algebraists. An element $x$ is called quasi-regular if there exists $y$ such that $x \circ y=y \circ x=0$. Recall that, for complex algebras, a nonzero complex number $\lambda$ is in the spectrum of $x$ provided $\lambda^{-1} x$ is not quasi-regular $[4$, p. 458]. We refer to [6] or [10] for the definition in the real case.

${ }^{4}$ The spectral radius is $r_{\mathfrak{n}}(x)=\max |\lambda|, \lambda \in \sigma_{\mathfrak{n}}(x)$.

5 This concept of regularity should not be confused with that introduced by von Neumann [8].

${ }^{6}$ It is obvious that the first definition always implies the second. In order to show that the second implies the first, it is sufficient to prove, for every closed set $F \subset{ } \boldsymbol{\Phi}$ r with $\phi_{\infty} \notin F$, the existence of $u \in \mathfrak{A}$ such that $u(\phi)=1$ for $\phi \in F$. This is, of course, trivial if $\mathscr{A}$ has an identity element. In case $\mathfrak{A}$ does not have an identity, the proof that such a $u$ exists, which seems to depend essentially on $\mathscr{A}$ being complex, will be found in [7, pp. 105-110]. 
with an identity element. In any case, if $\mathfrak{A}$ is regular, then it is normal in the following sense: If $F_{0}$ and $F_{1}$ are disjoint closed sets in $\Phi_{\mathfrak{I}}$ with $\phi_{\infty} \notin F_{1}$, then there exists $u \in \mathfrak{A}$ such that $u(\phi)=0$ for $\phi \in F_{0}$ and $u(\phi)=1$ for $\phi \in F_{1}$. The proof of this fact follows essentially the same lines as the proof given by Silov $[13$, p. 37] in the case considered by him. ${ }^{7}$

We turn now to the extension theorem mentioned in the introduction.

THEOREM 1. Let $\mathfrak{A}$ be a semi-simple regular Banach algebra which is algebraically embedded in a second Banach algebra $\mathfrak{B}$. Then every $\phi$ in $\Phi_{\mathfrak{X}}$ can be extended to an element of $\Phi_{\mathfrak{P}}$.

Proof. Let $\Psi$ denote the subset of $\Phi_{\mathfrak{a}}$ obtained by restricting elements of $\Phi_{\mathfrak{F}}$ to $\mathfrak{A}$. We have to prove $\Psi=\Phi_{\mathfrak{X}}$. Suppose that there exists an element $\phi_{0} \in \Phi_{\mathfrak{x}}-\Psi$. Then $\phi_{0} \neq \phi_{\infty}$ and, for every $\psi \in \Phi_{\mathfrak{O}}$, there exists $a_{\psi} \in \mathfrak{A}$ such that $a_{\psi}\left(\phi_{0}\right) \neq a_{\psi}(\psi)$. Set $\epsilon_{\psi}=\left|a_{\psi}(\psi)-a_{\psi}\left(\phi_{0}\right)\right| / 2$ and define the set

$$
G_{\psi}=\left\{\psi^{\prime}|| a_{\psi}\left(\psi^{\prime}\right)-a_{\psi}\left(\phi_{0}\right) \mid>\epsilon_{\psi}, \psi^{\prime} \in \Phi_{\mathfrak{B}}\right\} .
$$

Then $G_{\psi}$ is open in $\Phi_{\mathfrak{B}}$ and contains $\psi$. Since $\Phi_{\mathfrak{B}}$ is compact, there exists a finite number of sets $G_{\psi_{1}}, \cdots, G_{\psi_{n}}$ which cover $\Phi_{\mathscr{\vartheta}}$. Set $a_{i}=a_{\psi_{i}}, \epsilon=\min _{i} \epsilon_{\psi_{i}}$, and consider the neighborhood

$$
N_{0}=N_{\phi_{0}}\left(a_{1}, \cdots, a_{n} ; \epsilon\right)
$$

in $\Phi_{\mathfrak{x}}$. Then $N_{0}$ is disjoint from $\Psi$. Now choose in $\Phi_{\mathfrak{x}}$ an open set $G$ which contains $\phi_{0}$ and whose closure is contained in $N_{0}$. By the normality of $\mathfrak{A}$, there exist elements $u, v \in \mathfrak{A}$ such that $u\left(\phi_{0}\right)=1, u(\phi)=0$ for $\phi \notin G, v(\phi)=1$ for $\phi \in G$, and $v(\phi)=0$ for $\phi \notin N_{0}$. Then $(u v)(\phi)=u(\phi)$, for all $\phi \in \Phi_{\mathfrak{x}}$, and therefore $u v=u$ by the semisimplicity of $\mathfrak{A}$. Since $v(\psi)=0$ for every $\psi \in \Phi_{\mathfrak{F}}$, it follows that $v$ is quasi-regular in $\mathfrak{B}$. In other words, there exists $w \in \mathfrak{B}$ such that $v \circ w=0$. Observe that $u v=u$ implies $u \circ v=v$. Therefore $0=v \circ w$ $=(u \circ v) \circ w=u \circ(v \circ w)=u$. Since $u \neq 0$, this is a contradiction and completes the proof.

CoRollaRY 1. $\sigma_{\mathfrak{q}}(x)=\sigma_{\mathfrak{B}}(x)$ and hence $r_{\mathfrak{Y}}(x)=r_{\mathfrak{B}}(x)$ for every $x \in \mathfrak{A}$.

CoRollary 2. Let $\|x\|_{1}$ be any norm in $\mathfrak{A}$ under which $\mathfrak{A}$ is a (not necessarily complete) normed algebra. Then $r_{\mathfrak{I}}(x) \leqq\|x\|_{1}$ for all $x$.

If $\mathfrak{A}$ is taken to be the Banach algebra $C(\Omega)$ of all (real or complex) continuous functions vanishing at infinity on a locally compact

${ }^{7} \mathrm{~A}$ proof for the complex case without assumption of an identity is given by Mackey [7, pp. 105-110]. 
Hausdorff space $\Omega$, then $\mathfrak{A}$ is semi-simple and regular, and Corollary 2 reduces to the Kaplansky result mentioned in the introduction. The above proof of Theorem 1 was, in fact, suggested by the Kaplansky proof.

2. $B^{*}$-algebras. A complex Banach algebra $\mathfrak{A}$, not necessarily commutative or with an identity, is called a $B^{*}$-algebra [9] provided it has an involution $x \rightarrow x^{*}$ (a conjugate-linear anti-automorphism of period two) with the property $\left\|x x^{*}\right\|=\|x\|^{2}$ for every $x$. An algebra of type $C(\Omega)$ is a (commutative) $B^{*}$-algebra with involution $x \rightarrow x^{*}$ defined by

$$
x^{*}(\omega)=\overline{x(\omega)}, \quad \omega \in \Omega
$$

The important fact here is the converse $[1 ; 2]$. More precisely, every commutative $B^{*}$-algebra $\mathbb{S}$ is isometrically ${ }^{*}$-isomorphic to $C\left(\Phi_{\nwarrow}-\phi_{\infty}\right)$. In particular, commutative $B^{*}$-algebras are regular. Another consequence of this fact is that $\left\|x x^{*}\right\|=r\left(x x^{*}\right)$ for every $x$ in $\mathfrak{A}$. It follows directly from the algebraic properties of an involution that an element $x$ is quasi-regular if, and only if, each of the elements $x^{*}, x \circ x^{*}$, and $x^{*} \circ x$ is quasi-regular. Moreover, for every $x$, the spectrum of $x^{*}$ is equal to the complex conjugate

$$
\overline{\sigma_{\mathfrak{q}}(x)}
$$

of the spectrum of $x$.

In the remainder of our discussion, $\mathfrak{A}$ will be a $B^{*}$-algebra which is algebraically embedded in a general Banach algebra $\mathfrak{B}$. The norm in $\mathfrak{A}$ will be denoted by $\|x\|$ and the norm in $\mathfrak{B}$ by $\|x\|_{1}$. The theorem which follows is an extension of a previous result of the author's [9, Theorem 1.6].

THEOREM 2. In order for an element $x$ of $\mathfrak{A}$ to be quasi-regular in $\mathfrak{A}$ it is necessary and sufficient that both $x$ and $x^{*}$ be quasi-regular in $\mathfrak{B}$.

Proof. The necessity is obvious. Hence assume $x$ and $x^{*}$ to be quasi-regular in $\mathfrak{B}$. Then both $x \circ x^{*}$ and $x^{*} \circ x$ are quasi-regular in $\mathfrak{B}$. Let $\mathbb{S}$ be a maximal commutative $B^{*}$-subalgebra of $\mathfrak{A}$ which contains $x \circ x^{*}$, and let $\mathfrak{G}_{1}$ be a maximal commutative subalgebra of $\mathfrak{B}$ which contains $\mathfrak{S}$. Then both $\sigma \mathfrak{q}\left(x \circ x^{*}\right)=\sigma \mathfrak{s}\left(x \circ x^{*}\right)$ and $\sigma \mathfrak{C}_{1}\left(x \circ x^{*}\right)=\sigma_{\mathfrak{B}}\left(x \circ x^{*}\right)$. Moreover, since $\mathbb{E}$ is regular and is a subalgebra of $\mathfrak{E}_{1}$, it follows by Corollary 1 of Theorem 1 that $\sigma \Subset\left(x \circ x^{*}\right)$ $=\sigma_{\mathfrak{G}_{1}}\left(x \circ x^{*}\right)$. Therefore $\sigma \mathfrak{q}\left(x \circ x^{*}\right)=\sigma_{\mathfrak{g}}\left(x \circ x^{*}\right)$. This implies that $x \circ x^{*}$ is quasi-regular in $\mathscr{A}$. A similar argument shows that $x^{*} \circ x$ is also quasi-regular in $\mathfrak{A}$. Therefore $x$ must be quasi-regular in $\mathfrak{A}$, and the sufficiency is proved. 
Corollary 1. For every $x$ in $\mathfrak{A}$,

$$
\sigma_{\mathfrak{q}}(x)=\sigma_{\mathfrak{O}}(x) \cup \overline{\sigma_{\mathfrak{B}}\left(x^{*}\right)} .
$$

If $x$ is normal, then $\sigma \mathfrak{a}(x)=\sigma \circledast(x)$.

CoRollary 2. If the involution in $\mathcal{A}$ can be extended to an involution in $\mathfrak{B}$, then $x$ is quasi-regular in $\mathfrak{A}$ if, and only if, $x$ is quasi-regular in $\mathfrak{B}$. Hence, for every $x$ in $\mathfrak{A}, \sigma_{\mathfrak{q}}(x)=\sigma_{\mathfrak{\vartheta}}(x)$.

TheOREM 3. For every $x$ in $\mathfrak{A}$, it is true that

(i) $\|x\|^{2} \leqq\left\|x x^{*}\right\|_{1} \leqq\|x\|_{1}\left\|x^{*}\right\|_{1}$.

(ii) $r \mathfrak{a}(x)=r_{\mathfrak{B}}(x)$.

Proof. By Corollary 1 of Theorem 2, $\sigma_{\mathfrak{q}}\left(x x^{*}\right)=\sigma_{\mathfrak{F}}\left(x x^{*}\right)$, so that $r\left(x x^{*}\right)=r_{\mathscr{B}}\left(x x^{*}\right)$. Therefore

$$
\|x\|^{2}=\left\|x x^{*}\right\|=r_{\mathfrak{a}}\left(x x^{*}\right)=r_{\mathbb{B}}\left(x x^{*}\right) \leqq\left\|x x^{*}\right\|_{1} \leqq\|x\|_{1}\left\|x^{*}\right\|_{1},
$$

which proves (i). Now, for all $n$, we have $\left\|x^{n}\right\|\left\|^{2 / n} \leqq\right\| x^{n}\left\|_{1}^{1 / n}\right\|\left(x^{*}\right)^{n} \|_{1}^{1 / n}$ and, on letting $n \rightarrow \infty$, obtain $r q(x)^{2} \leqq r_{\mathfrak{B}}(x) r_{\mathscr{B}}\left(x^{*}\right)$. On the other hand, $r \mathfrak{q}(x)=r \mathfrak{q}\left(x^{*}\right)$ and always $r \mathfrak{F}(x) \leqq r \mathfrak{g}(x)$, since $\mathfrak{A}$ is a subalgebra of $\mathfrak{B}$. Therefore it follows that $r \boldsymbol{x}(x)=r_{\mathfrak{B}}(x)$.

CoROLlaRY. Let $\mathfrak{A}$ be an arbitrary $B^{*}$-algebra and let $\|x\|_{1}$ be any norm under which $\mathfrak{A}$ is a (not necessarily complete) normed algebra. Then $r a(x) \leqq\|x\|_{1}$ for every $x$. If, in addition, the second norm satisfies the condition $\left\|x^{*}\right\|_{1}=\|x\|_{1}$, then $\|x\| \leqq\|x\|_{1}$ for every $x$. If the second norm satisfies the condition $\left\|x x^{*}\right\|_{1}=\|x\|_{1}^{2}$, then ${ }^{9}\|x\|=\|x\|_{1}$ for every $x$.

\section{BIBLIOGRAPHY}

1. R. F. Arens, Representation of *algebras, Duke Math. J. vol. 14 (1947) pp. 269282.

2. I. Gelfand and M. Neumark, On the embedding of normed rings into the ring of operators in Hilbert space, Mat. Sbornik vol. 12 [54] (1943) pp. 197-213.

3. I. Gelfand, D. Raikov, and G. Silov, Commutative normed rings, Uspehi Mat. Nauk (N.S.) vol. 1 (1946) pp. 48-146.

4. E. Hille, Functional analysis and semi-groups, Amer. Math. Soc. Colloquium Publications, vol. 31, New York, 1948.

5. I. Kaplansky, Normed algebras, Duke Math. J. vol. 16 (1949) pp. 399-418.

6. - Topological rings, Bull. Amer. Math. Soc. vol. 54 (1948) pp. 809-826.

7. G. W. Mackey, Commutative Banach algebras (ed. by A. Blair), Harvard Lecture Notes, 1952.

8. J. von Neumann, On regular rings, Proc. Nat. Acad. Sci. U.S.A. vol. 22 (1936) pp. 707-713.

${ }^{8}$ An element $x$ is normal provided $x x^{*}=x^{*} x$. Observe that a normal element of $\mathscr{x}$ can be contained in a maximal commutative $B^{*}$-subalgebra of $\mathfrak{A}$.

- This was proved by Kaplansky [5, Theorem 6.4] for $B^{*}$-algebras and is an extension of a similar result for $C^{*}$-algebras due to Gelfand and Neumark [2]. 
9. C. E. Rickart, Banach algebras with an adjoint operation, Ann. of Math. vol. 47 (1946) pp. 528-550.

10. - The uniqueness of norm problem in Banach algebras, Ann. of Math. vol. 51 (1950) pp. 615-628.

11. I. E. Segal, The group algebra of a locally compact group, Trans. Amer. Math. Soc. vol. 61 (1947) pp. 69-105.

12. G. Silov, On the extension of maximal ideals, Doklady Acad. Sci. URSS. vol. 29 (1940) pp. 83-84.

13. - Regular normed rings, Trav. Inst. Math. Stekloff, vol. 21, Moscow, 1947.

YALE UNIVERSITY 